\title{
The efficacy of pharmacotherapy in postmenopausal osteoporosis: a longitudinal observational study
}

\author{
Wojciech Pluskiewicz'1, Piotr Adamczyk ${ }^{2}$, Edward Franek ${ }^{3}$, Ewa Sewerynek $k^{4}$, Hanna Wichrowska ${ }^{5}$, \\ Luiza Napiórkowska ${ }^{5}$, Michat Stuss' ${ }^{4}$, Aleksandra Rozwandowicz ${ }^{4}$, Bogna Drozdzowska ${ }^{6}$
}

${ }^{1}$ Department and Clinic of Internal Diseases, Diabetology, and Nephrology, Metabolic Bone Diseases Unit, Faculty of Medical Sciences in Zabrze, Medical University of Silesia, Katowice, Poland

${ }^{2}$ Department of Paediatrics, Faculty of Medical Sciences in Katowice, Medical University of Silesia in Katowice, Poland

${ }^{3}$ Mossakowski Medical Research Centre, Polish Academy of Sciences, Warsaw, Poland

${ }^{4}$ Department of Endocrine Disorders and Bone Metabolism, Medical University of Eódź, Poland

${ }^{5}$ Department of Internal Diseases, Endocrinology, and Diabetology, Central Clinical Hospital MSWiA, Warsaw, Poland

${ }^{6}$ Department and Chair of Pathomorphology, Faculty of Medical Sciences in Zabrze, Medical University

of Silesia, Katowice, Poland

\begin{abstract}
Introduction: The aim of the study was an assessment of longitudinal changes in fracture probability in postmenopausal women.

Material and methods: A group of 226 postmenopausal women at baseline mean age $66.46 \pm 7.96$ years were studied. There were 21 women without therapy, 102 taking calcium + vitamin D, and 103 women on antiresorptive therapy, in the study group. Data concerning clinical risk factors for osteoporosis and hip BMD were gathered. Fracture probability for major and hip fractures was established using FRAX ${ }^{\mathrm{TM}}$. Results: Mean follow-up time was $2.43 \pm 0.59$ years. Baseline FRAX value in the whole group for major fracture was $7.1 \pm 4.18$, and at follow-up it was 7.44 \pm 4.04 . Respective results for FRAX for hip fracture were $3.17 \pm 2.69$ and $3.02 \pm 2.35$. In the whole group the probability for major fractures significantly increased during follow-up $(p<0.05)$ and for hip fracture did not change. In non-treated patients and patients taking calcium + vitamin D the fracture probability increased significantly. In patients on antiresorptive therapy the fracture probability did not change, which was connected with an improvement in bone status assessed by DXA. Femoral neck T-score in the whole group did not change, in those not treated and taking calcium + vitamin D it decreased significantly $(p<0.05)$, while in treated women it increased significantly $(\mathrm{p}<0.05)$. In patients with improved bone status the FRAX values for major and hip fractures decreased by $0.44 \pm 1.62$ and $0.36 \pm 1.19$, respectively. Conversely, in patients with worsening T-score value the FRAX values increased by $1.33 \pm 1.42$ and $0.66 \pm 1.25$, respectively.

Conclusion: Antiresorptive therapy stabilises fracture probability in postmenopausal women due to improvement in bone status. (Endokrynol Pol 2019; 70 (6): 473-477)
\end{abstract}

Key words: antiresorptive therapy; bone status; follow-up; fracture probability; postmenopausal women

\section{Introduction}

The key problem in osteoporotic individuals is risk of fracture. Fractures are commonly the result of minimal trauma, e.g. a fall from standing height. Late consequences of fractures include limitations in functional status, and hip or spine fractures increase mortality. Osteoporotic fractures cause significant costs for health systems. One should remember that prior fracture increases the risk of subsequent fracture, being one of the most important clinical risk factors. Therefore, avoiding the first fracture should be a primary goal in the management of osteoporotic patients. Fracture risk depends on several clinical risk factors (age, sex, some medica- tions or underlying diseases) and bone status. Some of them are modifiable, the others are unmodifiable. Some years ago, a tool called FRAX for fracture risk assessment was developed by Kanis et al. [1]. FRAX combines some clinical risk factors and femoral neck BMD and is corrected by estimated lifetime, and the final result is a fracture probability. FRAX was developed in order to establish fracture probability in treatment-naive patients, and some thresholds for initiation of therapy were proposed. Fracture risk calculated by FRAX is used as a threshold for initiation of pharmacological therapy. In recent recommendations, FRAX values exceeding 3\% for hip fracture and $10 \%$ for major fracture are thresholds recommended for the onset of pharmacological 
therapy by Camacho et al. [2]. After some discussion and with the awareness of limitations of FRAX [3, 4], the same thresholds were proposed in Poland by Lorenc et al. [5], and they remain valid [6]. One may expect that baseline fracture probability in efficiently treated patients should diminish or stabilise at follow-up. In several studies medications used in osteoporosis decrease fracture incidence, so one may expect that also fracture probability should decrease. In our previous study in a retrospective analysis performed in a group of 191 postmenopausal women, we proved that antiresorptive therapy may be considered as profitable for skeletal status, as was shown by Puskiewicz et al. [7]. In previously mentioned recommendations the change in longitudinal FRAX values was one of the criteria of therapeutic efficacy [2].

In the current study we analysed in a longitudinal observation whether therapy for osteoporosis is able to diminish fracture probability expressed by FRAX.

\section{Material and methods}

In the study a group of 226 postmenopausal women were observed. They were recruited in Polish outpatient osteoporotic clinics in Warsaw ( $\mathrm{n}=88)$, Łódź $(\mathrm{n}=79)$, and Zabrze $(\mathrm{n}=59)$. All women were treatment naive at baseline. Data on clinical risk factors necessary for the calculation of fracture probability according to FRAX were collected at baseline and follow-up. At baseline in 101 patients no clinical risk factors were present, one risk factor was present in 88, two in 29 , three in seven, and four risk factors were present in one woman. Seventy-nine (34.9\%) women reported at enrolment that they had experienced at least one osteoporotic fracture previously. Twenty-four of them had had multiple fractures (two fractures were reported by 15 subjects, three fractures by eight subjects, and four fractures by one woman).

Bone status at the proximal femur was established using Hologic Explorer (Zabrze) and Lunar GE (Warsaw and Łódź) densitometers at baseline and at follow-up. Precision of measurements was established separately for the above-mentioned DXA devices and expressed as root-mean-square coefficient of variation (CV\%). CV\% values calculated for Zabrze, Warsaw, and Łódź were: $1.83 \%, 1.48 \%$, and $0.90 \%$, respectively.

Fracture probability for hip and major fractures was established using the Polish version of FRAX.

Each patient received therapeutic recommendations from their physician according to individual assessment.

Table I shows the clinical characteristics of the studied women. Among them were 21 patients without therapy (subgroup 1), 102 taking calcium + vit. D (subgroup 2), and 103 on antiresorptive therapy and calcium + vitamin D (subgroup 3 ). The most common medication used was ibandronic acid $(\mathrm{n}=43)$ followed by alendronate $(n=28)$ and risedronate $(n=25)$. Generally, the medications did not change in the duration of the study.

The mean age did not differ among subgroups.

Local Ethics Committees approved the study protocols, and each woman gave written, informed consent.

\section{Results}

Mean follow-up was $2.43 \pm 0.59$ years in the whole group. Mean follow-up in subgroups 1, 2, and 3 were $2.08 \pm 0.31,2.49 \pm 0.61$, and $2.43 \pm 0.6$ years, respectively. Follow-up was significantly shorter in the untreated women than in the other subgroups $(p<0.01)$ and did not differ between subgroups 2 and 3 . The number of clinical risk factors did not change significantly during follow-up (data not shown).

Table II includes the results for fracture probability for major and hip fractures in the whole group and in subgroups. The baseline fracture probability differed significantly between subgroups, being the lowest in the untreated women and the highest in those receiving antiresorptive treatment. The same obvious trend concerns bone densitometry results. In regard to longitudinal changes in the whole group, the fracture probability increased for major fracture $(p<0.01)$, and the hip fracture probability did not change. In untreated women major and hip fractures probability increased

Table I. Clinical characteristic of women studied - all women and subgroups (mean, SD)

\begin{tabular}{|c|c|c|c|c|}
\hline & $\begin{array}{c}\text { Whole } \\
\text { group } \\
\text { (n= 226) }\end{array}$ & $\begin{array}{c}\begin{array}{c}\text { Untreated } \\
\text { women } \\
\text { Subgroup } 1(n=21)\end{array} \\
\end{array}$ & $\begin{array}{c}\text { Women on calcium } \\
\text { and vitamin } D \\
\text { Subgroup } 2 \text { ( } n=102 \text { ) }\end{array}$ & $\begin{array}{l}\text { Women on } \\
\text { antiresorptive therapy } \\
\text { Subgroup } 3(n=103)\end{array}$ \\
\hline Age [years] at baseline & $66.6 \pm 7.96$ & $67.3 \pm 7.1$ & $66.4 \pm 7.3$ & $66.5 \pm 8.8$ \\
\hline Age [years] at follow-up & $69.0 \pm 8.02$ & $69.4 \pm 7.1$ & $68.9 \pm 7.3$ & $69.0 \pm 8.9$ \\
\hline Weight [kg] at baseline & $67.35 \pm 12.6$ & $75.0 \pm 15.3$ & $69.7 \pm 11.8$ & $63.5 \pm 11.6$ \\
\hline Weight [kg] at follow-up & $67.7 \pm 13.2$ & $74.6 \pm 15.6$ & $70.2 \pm 12.2$ & $63.9 \pm 12.5$ \\
\hline Height $[\mathrm{cm}]$ at baseline & $158.1 \pm 5.9$ & $160.4 \pm 5.0$ & $158.4 \pm 5.6$ & $157.3 \pm 6.2$ \\
\hline Height [cm] at follow-up & $157.6 \pm 5.9^{*}$ & $160.1 \pm 5.2$ & $158.0 \pm 5.7^{*}$ & $156.7 \pm 6.2^{* *}$ \\
\hline BMI $\left[\mathrm{kg} / \mathrm{m}^{2}\right]$ at baseline & $26.9 \pm 4.9$ & $29.2 \pm 6.0$ & $27.8 \pm 4.7$ & $25.6 \pm 4.5$ \\
\hline BMI $\left[\mathrm{kg} / \mathrm{m}^{2}\right]$ at follow-up & $27.3 \pm 5.1^{\wedge}$ & $29.1 \pm 6.2$ & $28.1 \pm 4.9 \wedge \wedge$ & $26.1 \pm 4.9^{\wedge} \wedge$ \\
\hline Previous fracture at baseline & 79 (34.9\%) & $2(9.5 \%)$ & $29(28.4 \%)$ & $48(46.6 \%)$ \\
\hline
\end{tabular}

$\mathrm{BMI}$ - body mass index; ${ }^{*}$ significantly lower than at baseline, $\mathrm{p}<0.0001 ;{ }^{* *}$ significantly lower than at baseline, $\mathrm{p}<0.01 ; \wedge$ significantly higher than at baseline $\mathrm{p}<0.01 ; \wedge \wedge$ significantly higher than at baseline, $\mathrm{p}<0.05$ 
Table II. Results for fracture probability according to FRAX for major and hip fractures and for FN T-scores in the whole group and in subgroups at baseline and follow-up (mean, SD). Differences between subgroups and between baseline and follow-up are shown

\begin{tabular}{|c|c|c|c|c|}
\hline & $\begin{array}{c}\text { Whole } \\
\text { group } \\
\text { (n= 226) }\end{array}$ & $\begin{array}{c}\begin{array}{c}\text { Untreated } \\
\text { women }\end{array} \\
\text { Subgroup } 1(n=21)\end{array}$ & $\begin{array}{l}\text { Women on calcium } \\
\text { and vitamin } D \\
\text { Subgroup } 2 \text { ( } n=102 \text { ) }\end{array}$ & $\begin{array}{l}\text { Women on } \\
\text { antiresorptive therapy } \\
\text { Subgroup } 3(n=103 \text { ) }\end{array}$ \\
\hline Major fracture at baseline (\%) & $7.1 \pm 4.18$ & $3.88 \pm 1.4^{*}$ & $6.05 \pm 2.88^{@}$ & $8.79 \pm 4.87$ \\
\hline Major fracture at follow-up (\%) & $7.44 \pm 4.04$ & $4.3 \pm 1.65^{*}$ & $6.61 \pm 2.93^{@}$ & $8.91 \pm 4.7$ \\
\hline p value ${ }^{\#}$ & 0.01 & 0.05 & 0.0001 & NS \\
\hline Hip fracture at baseline (\%) & $2.16 \pm 2.23$ & $0.63 \pm 0.52^{* *}$ & $1.46 \pm 1.31 @$ & $3.17 \pm 2.69$ \\
\hline Hip fracture at follow-up (\%) & $2.25 \pm 2.04$ & $0.84 \pm 0.72^{* *}$ & $1.76 \pm 1.53^{@}$ & $3.02 \pm 2.35$ \\
\hline p value ${ }^{\#}$ & NS & 0.01 & 0.0001 & NS \\
\hline FN T-score at baseline & $-1.71 \pm 0.81$ & $-0.85 \pm 0.76^{* * *}$ & $-1.48 \pm 0.67 @ @$ & $-2.13 \pm 0.71$ \\
\hline FN T-score at follow-up & $-1.74 \pm 0.72$ & $-1.02 \pm 0.74^{* * *}$ & $-1.58 \pm 0.63 @ @$ & $-2.05 \pm 0.63$ \\
\hline p value ${ }^{\#}$ & NS & 0.05 & 0.05 & 0.05 \\
\hline
\end{tabular}

NS - non significant; " for comparison between follow-up and baseline examination; * significantly lower than in women on calcium + vitamin D supplementation $(\mathrm{p}<0.001)$ and in women on antiresorptive therapy $(\mathrm{p}<0.0001)$; ** significantly lower than in women on calcium + vitamin $\mathrm{D}$ supplementation $(\mathrm{p}<0.01)$ and in women on antiresorptive therapy $(p<0.0001) ;{ }^{* * *}$ significantly higher than in women on calcium + vitamin $D$ supplementation $(p<0.001)$ and in women on antiresorptive therapy $(\mathrm{p}<0.0001)$; @Significantly lower than in women on antiresorptive therapy $(\mathrm{p}<0.0001)$; @@significantly higher than in women on antiresorptive therapy $(p<0.0001)$

( $\mathrm{p}<0.05$ and $<0.01$, respectively). Also, in subgroup 2 both major and hip fracture probability increased $(\mathrm{p}<0.0001)$. In subgroup 3 the FRAX values did not change significantly.

Femoral neck (FN) T-score in the whole group did not change. In subgroups 1 and 2 it decreased significantly ( $p<0.05)$, and in subgroup 3 it increased significantly from $-2.13 \pm 0.71$ to $-2.05 \pm 0.63(\mathrm{p}<0.05)$ (Tab. II). In 127 (56\%) women the T-score increased, and in $99(44 \%)$ it decreased during follow-up. In patients with improved bone status the FRAX values for major and hip fractures decreased by $0.44 \pm 1.62$ and $0.36 \pm 1.19$, respectively. Conversely, in patients with worse Tscore the FRAX values increased by $1.33 \pm 1.42$ and $0.66 \pm 1.25$, respectively. A comparison of longitudinal changes in FRAX values in these subgroups $(n=127$ and $n=99)$ shows significant differences $(\mathrm{p}<0.0001)$.

Figures 1 and 2 present correlations between longitudinal changes in FRAX and FN T-score. For both fracture probability variables an improvement was significantly connected with an improvement in bone status.

During follow-up eight fractures were observed (four in patients with prior fracture and four in women without prior fracture), but due the small number of fractures this factor was not analysed. Those new fractures were recognised in the forearm (3), spine (2), and other skeletal sites (3).

\section{Discussion}

The most important finding of the current longitudinal study is the observation that in women treated with antiresorptive agents the fracture probability did not change during the period of observation. Adequate data in other patients not receiving the antiresorptive therapy showed an increase in fracture probability. We consider that the current observations support the efficacy of pharmacological management and are important for practitioners. The improvement in fracture probability was connected with the positive influence of patients' management on bone status. Increasing age negatively influences fracture probability, so even in a case of stable BMD, the FRAX value will increase. Such an observation of positive influence on bone status and secondary stabilisation in fracture probability clearly indicates the main direction in management. One may hypothesise that fracture probability would change also when the number of clinical risk factors changes. In the current study we did not observe longitudinal changes in regard to the number of clinical risk factors. Therefore, the only factor positively influencing fracture probability was an improvement in bone status. Current results are consistent with our previous data obtained in retrospective analysis in a group of 191 women [7]; in this study the correlation between changes in FRAX for major fracture with changes in T-score was slightly stronger than in the current study $(-0.76$ vs. -0.66 , $\mathrm{p}<0.05)$ while FRAX for hip fracture was almost the same (0.631 vs. 0.613 , non-significant). We consider that a positive influence on bone status was responsible for a good response to treatment measured by the level of fracture probability. These observations are clearly visible in Figures 1 and 2. 


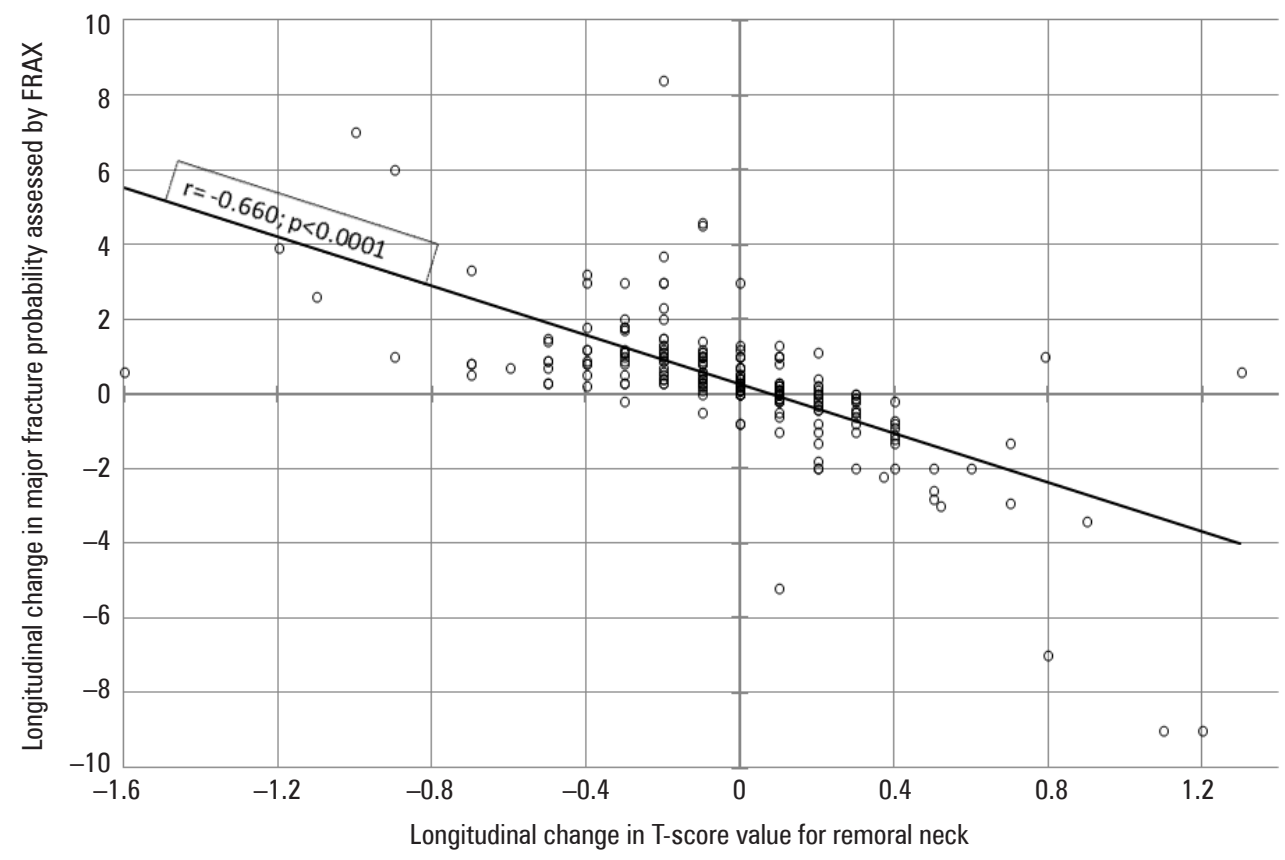

Figure 1. Relationships between change in major fracture probability assessed by FRAX and change in T-score value for femoral neck during the follow-up period

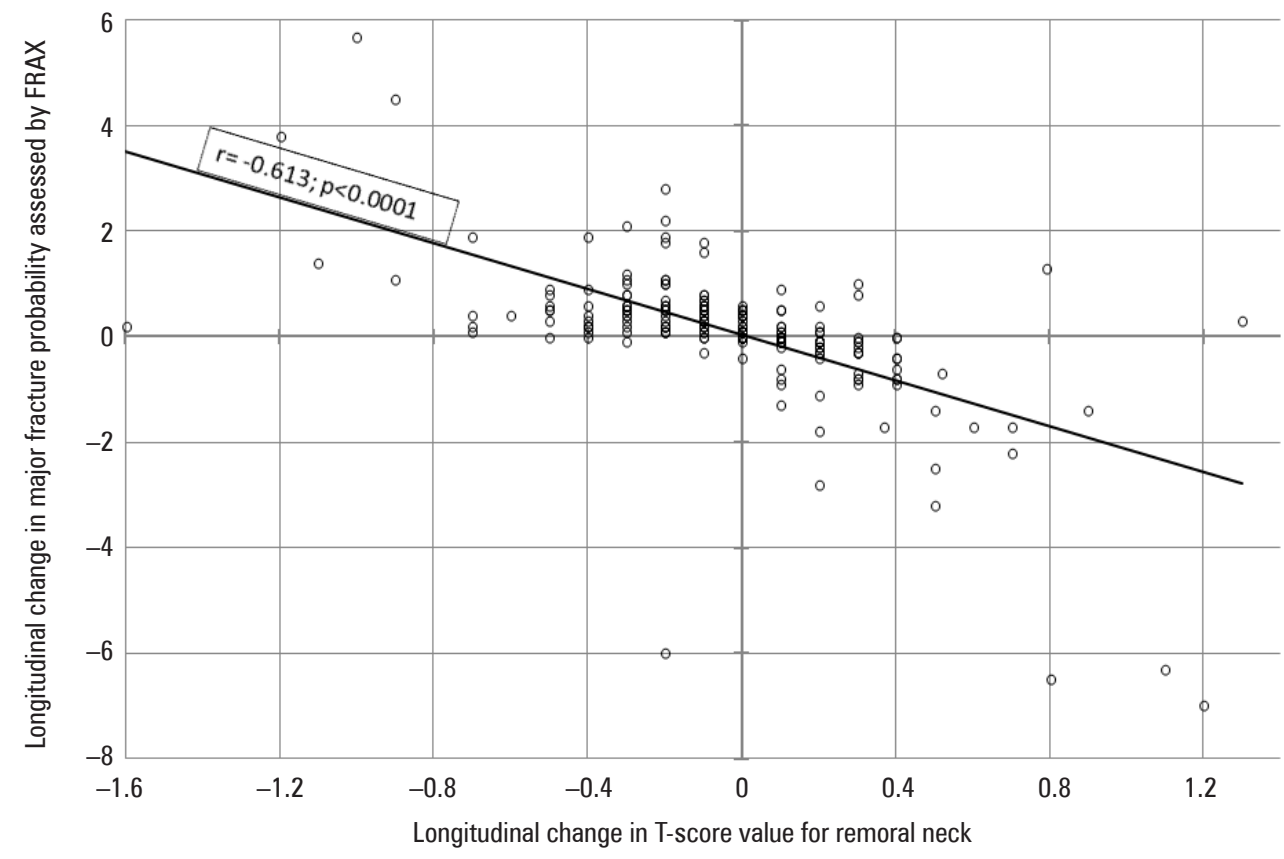

Figure 2. Relationships between change in hip fracture probability assessed by FRAX and change in T-score value for femoral neck during the follow-up period

In a longitudinal four-year study 11,049 untreated women aged over 50 years were given antiosteoporotic therapy by Leslie et al. [8]. Information about clinical risk factors for osteoporosis were gathered along with BMD measurements. At baseline, FRAX for major fracture was $8.2 \%$ and increased to $9.6 \%$ at the final point. Respective data for hip were $1.1 \%$ and $1.6 \%$, respectively. We noted in patients treated by antiresorptive medication only a small, non-significant increase in FRAX for major fracture and a non-significant decrease for hip FRAX (Tab. II). In the cited study the FN T-score decreased from -1.6 to -1.7 , and we observed a small but significant increase. Similarly to the current study, the authors did not observe changes in the number of clinical risk factors. Probably the longer follow-up in comparison to our study revealed a stronger influence of ageing. 
Of course, the most important point concerns fracture incidence in a longitudinal observation. In the current study the number of new fractures was too small, so this criterion cannot be verified, and further follow-up should take into consideration the fracture incidence. We should also remember that patients were non-randomly allocated into subgroups in regard to the kind of management. Due to such pattern of patients' allocation to the analyzed groups, the study design may be considered more adhere to everyday practice. Therefore, the results of the current observation have significance for practitioners. One might expect that the FRAX algorithm would properly predict fracture incidence. In a study performed in 815 Japanese women by Tamaki et al. [9] a 10-year follow-up observed that the fracture rate did not differ significantly from that predicted by FRAX [9]. FRAX was also compared with other screening tools to identify women with increased risk of fracture [10, 11]. In a population-based, prospective study performed in Denmark by Rubin et al. [10], comprising 3614 women, FRAX did not give better fracture risk prediction than several different screening methods (OST, ORAI, OSIRIS, SCORE, and age alone). Also, a fragility index had predictive power comparable to FRAX in women from the Global Longitudinal Study in Women (GLOW) [11]. Another algorithm for fracture prediction - QFractureScores by Hippisley-Cox et al. [12] — was evaluated in a prospective study by Collins et al. [13]. This study showed predictive power for fracture for QFracturesScores.

Our study has some limitations. The number of subjects studied and duration of follow-up did not allow us to gather a sufficient number of fractures necessary to prove longitudinal efficacy in regard to fracture incidence. In particular, the number of women without therapy was small. During the study, the treatment recommendations for patients enrolled in each of the three participating centers could have been different, depending on the physicians' experience and preferences.

The follow-up was significantly shorter in the untreated women than in other subgroups. We consider that further analysis performed in larger groups with longer follow-up will establish both stabilisation in fracture probability and a decrease in fracture incidence.

\section{Conclusion}

Antiresorptive therapy stabilises fracture probability in postmenopausal women due an improvement in bone status.

\section{References}

1. Kanis JA, Johnell O, Oden A, et al. FRAX and the assessment of fracture probability in men and women from the UK. Osteoporos Int. 2008; 19(4): 385-397, doi: 10.1007/s00198-007-0543-5, indexed in Pubmed: 18292978.

2. Camacho PM, Petak SM, Binkley N, et al. American Association of Clinical Endocrinologists and American College of Endocrinology Clinical Practice Guidelines for the Diagnosis and Treatment of Postmenopausal Osteoporosis - 2016. Endocr Pract. 2016; 22(Suppl 4): 1-42, doi: 10.4158/EP161435.GL, indexed in Pubmed: 27662240.

3. Marcinkowska M, Ignaszak-Szczepaniak M, Wawrzyniak A, et al. A discussion of the intervention thresholds in osteoporosis treatment in Poland. Endokrynol Pol. 2011; 62(1): 30-36, indexed in Pubmed: 21365576.

4. Franek $\mathrm{E}$, Wichrowska $\mathrm{H}$, Gozdowski $\mathrm{D}$, et al. WHO fracture risk calculator (FRAX) in the assessment of obese patients with osteoporosis. Endokrynol Pol. 2009; 60(2): 82-87, indexed in Pubmed: 19396750.

5. Lorenc RS, Głuszko P, Karczmarewicz E, et al. [Diagnostic and therapeutic recommendations in osteoporosis] (in Polish). Medycyna Praktyczna Reumatologia. 2013; 1(special issue): 1-44.

6. Lorenc R, Głuszko P, Franek E, et al. Guidelines for the diagnosis and management of osteoporosis in Poland : Update 2017. Endokrynol Pol. 2017; 68(5): 604-609, doi: 10.5603/EP.2017.0062, indexed in Pubmed: 29168548.

7. Pluskiewicz W, Drozdzowska B, Adamczyk P. Ten-year fracture risk in the assessment of osteoporosis management efficacy in postmenopausal women: a pilot study. Climacteric. 2013; 16(1): 117-126, doi: 10.3109/136 97137.2011.646345, indexed in Pubmed: 22335356.

8. Leslie WD, Majumdar SR, Lix LM, et al. Can change in FRAX score be used to "treat to target"? A population based cohort study. J Bone Miner Res. 2014; 29(5): 1074-1080, indexed in Pubmed: 24877235.

9. Tamaki J, Iki M, Kadowaki E, et al. Fracture risk prediction using FRAX®: a 10-year follow-up survey of the Japanese Population-Based Osteoporosis (JPOS) Cohort Study. Osteoporos Int. 2011; 22(12): 3037-3045, doi: 10.1007/s00198-011-1537-x, indexed in Pubmed: 21279504.

10. Rubin $\mathrm{KH}$, Abrahamsen Bo, Friis-Holmberg T, et al. Comparison of different screening tools (FRAX®, OST, ORAI, OSIRIS, SCORE and age alone) to identify women with increased risk of fracture. A population-based prospective study. Bone. 2013; 56(1): 16-22, doi: 10.1016/j. bone.2013.05.002, indexed in Pubmed: 23669650.

11. Li G, Thabane L, Papaioannou A, et al. Comparison between frailty index of deficit accumulation and fracture risk assessment tool (FRAX) in prediction of risk of fractures. Bone. 2015; 77: 107-114, doi: 10.1016/j bone.2015.04.028, indexed in Pubmed: 25916552.

12. Hippisley-Cox J, Coupland C. Predicting risk of osteoporotic fracture in men and women in England and Wales: prospective derivation and validation of QFractureScores. BMJ. 2009; 339: b4229, doi: 10.1136/bmj. b4229, indexed in Pubmed: 19926696.

13. Collins GS, Mallett S, Altman DG. Predicting risk of osteoporotic and hip fracture in the United Kingdom: prospective independent and external validation of QFractureScores. BMJ. 2011; 342: d3651, doi: 10.1136/bmj. d3651, indexed in Pubmed: 21697214. 